\title{
Plasma flow reactor for steady state monitoring of physical and chemical processes at high temperatures
}

Cite as: Rev. Sci. Instrum. 88, 093506 (2017); https://doi.org/10.1063/1.5001346

Submitted: 17 May 2017 . Accepted: 22 August 2017 . Published Online: 11 September 2017

Batikan Koroglu, Marco Mehl, Michael R. Armstrong, Jonathan C. Crowhurst, David G. Weisz, Joseph M. Zaug (D), Zurong Dai, Harry B. Radousky, Alex Chernov, Erick Ramon, Elissaios Stavrou, Kim Knight, Andrea L. Fabris, Mark A. Cappelli, and Timothy P. Rose

\section{ARTICLES YOU MAY BE INTERESTED IN}

Formation of ${ }^{238} \mathrm{U}^{16} \mathrm{O}$ and ${ }^{238} \mathrm{U}^{18} \mathrm{O}$ observed by time-resolved emission spectroscopy subsequent to laser ablation

Applied Physics Letters 111, 034101 (2017); https://doi.org/10.1063/1.4991824

Field-reversed configuration formed by in-vessel $\theta$-pinch in a tandem mirror device

Review of Scientific Instruments 88, 093505 (2017); https://doi.org/10.1063/1.5001313

Spatially resolved spectroscopic ion temperature measurements at plasma edge of the T-10 tokamak

Review of Scientific Instruments 88, 093508 (2017); https://doi.org/10.1063/1.5001490

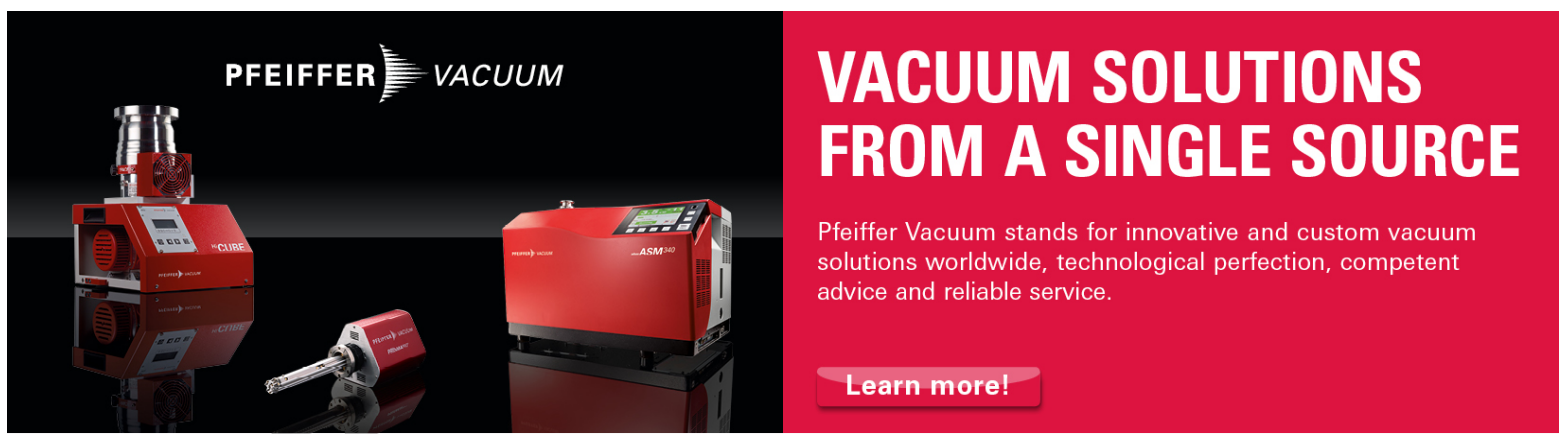




\title{
Plasma flow reactor for steady state monitoring of physical and chemical processes at high temperatures
}

\author{
Batikan Koroglu, ${ }^{1, a)}$ Marco Mehl, ${ }^{1}$ Michael R. Armstrong, ${ }^{1}$ Jonathan C. Crowhurst, ${ }^{1}$ \\ David G. Weisz, ${ }^{1}$ Joseph M. Zaug, ${ }^{1}$ Zurong Dai, ${ }^{1}$ Harry B. Radousky, ${ }^{1}$ Alex Chernov, ${ }^{1}$ \\ Erick Ramon, ${ }^{1}$ Elissaios Stavrou, ${ }^{1}$ Kim Knight, ${ }^{1}$ Andrea L. Fabris, ${ }^{2, b)}$ \\ Mark A. Cappelli, ${ }^{2}$ and Timothy P. Rose ${ }^{1}$ \\ ${ }^{1}$ Physical and Life Sciences Directorate, Lawrence Livermore National Laboratory, Livermore, \\ California 94550, USA \\ ${ }^{2}$ Department of Mechanical Engineering, Stanford University, Stanford, California 94305, USA
}

(Received 17 May 2017; accepted 22 August 2017; published online 11 September 2017)

\begin{abstract}
We present the development of a steady state plasma flow reactor to investigate gas phase physical and chemical processes that occur at high temperature $(1000<\mathrm{T}<5000 \mathrm{~K})$ and atmospheric pressure. The reactor consists of a glass tube that is attached to an inductively coupled argon plasma generator via an adaptor (ring flow injector). We have modeled the system using computational fluid dynamics simulations that are bounded by measured temperatures. In situ line-of-sight optical emission and absorption spectroscopy have been used to determine the structures and concentrations of molecules formed during rapid cooling of reactants after they pass through the plasma. Emission spectroscopy also enables us to determine the temperatures at which these dynamic processes occur. A sample collection probe inserted from the open end of the reactor is used to collect condensed materials and analyze them ex situ using electron microscopy. The preliminary results of two separate investigations involving the condensation of metal oxides and chemical kinetics of high-temperature gas reactions are discussed. Published by AIP Publishing. [http://dx.doi.org/10.1063/1.5001346]
\end{abstract}

\section{INTRODUCTION}

Gas phase chemical and physical transformations occurring at high-temperatures $(1000-5000 \mathrm{~K})$ on millisecond to sub-second time scales are relevant to a broad range of scientific and technical applications. Inductively coupled plasma (ICP) generation ${ }^{1}$ offers a well-characterized experimental approach for investigating these processes. Common ICP applications include high sensitivity trace element measurements, ${ }^{2}$ the investigation of fundamental properties of plasmas, ${ }^{3}$ and material synthesis. ${ }^{4,5}$ However, this method can also be useful for studying gas phase kinetics of recombination reactions as well as slow processes such as condensation, provided that a flow tube reactor is coupled to the ICP generator to increase the residence time of the reactants. Therefore, in this study, a glass tube is connected to the quartz torch of an ICP generator to dissociate the molecules into their constituent atoms and to study the subsequent chemical and physical processes at high temperatures.

Flow reactors provide residence times ranging from milliseconds to a few seconds depending on the tube dimensions and flow rates. ${ }^{6}$ Other experimental platforms such as shock tubes enable the study of faster processes (e.g., combustion reactions) that occur within hundreds of microseconds. ${ }^{7,8}$ The combined use of inductively coupled plasma and flow reactor generates a continuous, steady flow of reactants, which has advantages including (1) high signal to noise ratio (low signal

\footnotetext{
a)Author to whom correspondence should be addressed: koroglu1@1lnl.gov b)Present address: Surrey Space Centre, University of Surrey, Guildford, United Kingdom.
}

intensities can be integrated over long times), (2) flexibility in controlling the distribution of analytes, (3) ability to simultaneously observe qualitatively disparate phenomena, such as chemical kinetics and condensation processes, over several orders of magnitude in time, (4) in situ investigation of high temperature processes using optical spectroscopy techniques (e.g., emission and absorption spectroscopy), and (5) ex situ analysis of reaction products using electron microscope techniques [e.g., scanning electron microscope (SEM) and transmission electron microscope (TEM)]. In addition, since the states of interest are continuously generated, time dependent diagnostics (such as short pulse lasers) are not necessary-the observation of time dependent effects is implicit in the design of the instrument.

Here, we discuss the development and testing of an ICP generator coupled with a flow reactor, hereafter referred to as the plasma flow reactor. The heat transfer and fluid flow characteristics of the system are described by computational fluid dynamics simulations and constrained by temperature measurements. Preliminary results from experiments using two types of reactants are presented. The first uses aqueous metal salt solutions $(\mathrm{Fe}, \mathrm{Al})$ to study the condensation and growth of oxide particles. The second uses a dilute hydrocarbon gas (acetylene) to evaluate the kinetics of decomposition and recombination reactions.

\section{DESIGN FEATURES OF THE PLASMA FLOW REACTOR}

A commercially available ICP optical emission spectrometer (Profile Model, Teledyne Leeman Instruments) was 


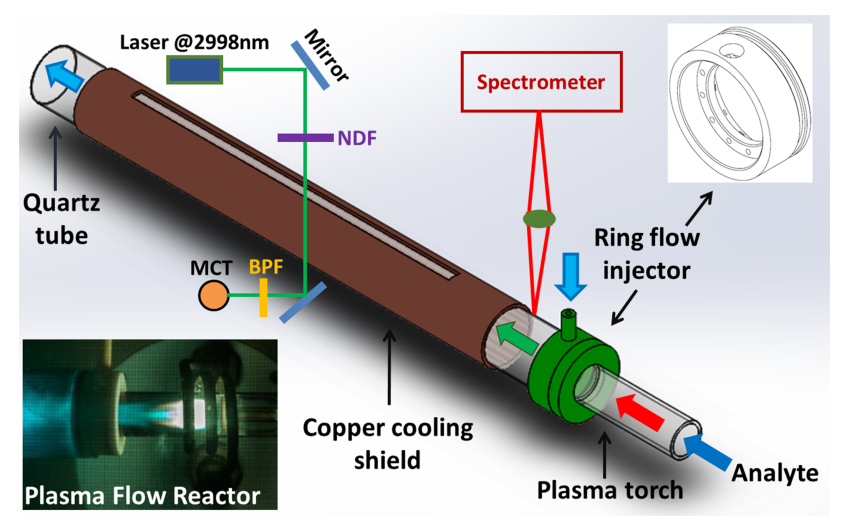

FIG. 1. The schematic of the plasma flow reactor experimental setup. (NDF: neutral density filter, MCT: Mercury Cadmium Telluride detector, BPF: band pass filter).

modified by removing the original spectrometer and attaching a $1 \mathrm{~m}$ long quartz tube ( $37 \mathrm{~mm}$ ID, $40 \mathrm{~mm}$ OD) to the plasma torch outlet (20 mm OD) using an adapter (ring flow injector) shown in Fig. 1. The ring flow injector is designed to allow radial flow of gas into the quartz tube via twelve $1.6 \mathrm{~mm}$ OD holes oriented parallel to the flow reactor axis. The inset of Fig. 1 shows its design. Gas flow from the ring flow injector creates a flow stream around the high-temperature core, preventing the melting of the quartz tube. Depending on the flow conditions, it also permits mixing and cooling rates to be adjusted within the reactor tube. The flow rate through the ring flow injector is varied via a digital flow controller (Omega, FMA5528A).

The quartz tube serves two important purposes. First, it enables the in situ investigation of chemical processes that occur as the plasma gas cools. Optical spectroscopy techniques (emission and absorption) are used to measure both chemical species and temperatures at various positions along the reactor axis so that the chemical evolution of the system can be monitored. The details of the spectrometer for emission measurements and the laser setup are explained in Secs. III B and IV B, respectively. The quartz tube also allows the physical collection of particles using a narrow diameter probe $(6 \mathrm{~mm}$ OD) that is inserted from the open end of the tube. A moving stage is vertically positioned next to the plasma flow reactor to accommodate the optics and other measurement tools such as the particle collection probe. A copper shield envelops the quartz tube to maintain its external wall temperature at $20^{\circ} \mathrm{C}$ through an in-house chiller that circulates water through a tube (not shown in the figure) brazed to the shield. The copper shield was slotted at the top and bottom to enable line of sight laser absorption and/or optical emission measurements. Note that the diameter of the flow reactor as well as the flow rates can be changed to vary chemical species residence times along the reactor. For the $37 \mathrm{~mm}$ ID reactor tube used in our experiments, a residence time of about $10 \mathrm{~ms}$ is obtained over $1 \mathrm{~cm}$ distance when the temperature and flow rate of the argon gas flowing through the reactor are $1000 \mathrm{~K}$ and $40 \mathrm{l} / \mathrm{min}$, respectively.

The plasma torch consists of a Radio-Frequency (RF) inductively coupled plasma generation system operating at $40 \mathrm{MHz}$. This type of plasma source has been extensively developed and characterized as described in the literature targeting a broad spectrum of applications where high temperature flow fields are required. ${ }^{1}$ The neutral gas temperature in the plasma core lies in the range of 3000-10 $000 \mathrm{~K}$, depending on the source design, operating gas and power. ${ }^{1}$ The plasma used here is generated by a 4-loop inductive antenna wrapped around a $20 \mathrm{~mm}(\mathrm{OD})$ axial quartz torch. The quartz torch contains three coaxial channels, with 18,14 , and $1.6 \mathrm{~mm}$ (ID) cross section diameters, respectively. The outermost flow provides a flow barrier between the plasma and the quartz wall to reduce the plasma heat flux and avoid softening or melting of the outer tube. The central flow serves as a primary gas (generally argon) supply to the plasma. The innermost flow is used to inject other gases or substances into the high temperature core. Selection of relative flow rates to these three channels is dependent on the specific application. Three different digital flow controllers were used to adjust the flow rates of the outermost flow (Omega, FMA5526A), central flow (Omega, FMA5518A), and innermost flow (Omega, FMA2605A).

The RF generator is based on a triode tube amplifier and a LC-parallel resonant circuit that determines and drives the operating frequency. The system was modified to be powered separately by a PK series Glassman high voltage (HV) DC power supply, allowing the power of the system to be adjusted between 700 and $1400 \mathrm{~W}$. For most of the experimental work described in this paper, the operating conditions were around $750 \mathrm{~W}$. The triode filament is heated by applying $60 \mathrm{~Hz} \mathrm{AC}$ power directly from the main power line using a variable transformer to match the filament current requirement. Both the triode oscillator tube and the antenna are water-cooled for long-term operation. A piezoelectric spark igniter is used to trigger the discharge.

\section{SYSTEM CHARACTERIZATION}

The design and performance of the plasma flow reactor were characterized through a combination of computational fluid dynamics modeling and temperature measurements. Preliminary calculations were used to optimize the system geometry and ring flow injector design to achieve the desired residence times and cooling rates. Experimental temperature measurements provided validation data for the model and improved its tuning. The resulting model provides constraints on the flow path and thermal history of the materials injected into the reactor for different operating conditions.

\section{A. Computational fluid dynamics model}

The heat transfer and fluid flow characterization of the reactor involved both 2D and 3D multi-physics calculations using the Star CCM+ fluid dynamics code. The numerical model is conveniently split into two distinct computational domains, which can be solved in sequence: (1) the plasma torch, where the plasma is generated and (2) the reactor tube, where the atomized precursors recombine to form gas phase products and particles. The temperature and flow field of the plasma torch were estimated using 2D steady axisymmetric calculations. This was allowable due to the short residence time, relatively high local velocities, absence of backflow, and limited feedback at the exit of the plasma nozzle. In contrast, 
when gas expands into the reaction tube and cools through a combination of radiative, convective, and conductive heat loss, the intrinsically long residence times and steep temperature gradients lead to thermal stratification that requires full 3D calculations to represent the flow field and temperature distribution.

The plasma torch was simulated imposing a homogeneous heat source to represent the ohmic heating of the argon gas induced by the RF field. Temperature and flow profiles obtained with this simplified approach are consistent with what is reported in the literature ${ }^{9}$ for similar setups. The power of the heat source was initially based on the RF power supply readings and later adjusted based on the temperature measurements discussed in the following paragraphs. The temperature and velocity profiles obtained at the end of the torch section were imposed as boundary conditions to the reactor tube section. Due to the high dilution of the analyte relative to the carrier gas, fluid properties are assumed to be those of argon. Temperature dependent viscosities and thermal conductivities were fitted to literature data, ${ }^{10,11}$ density is assumed to follow the ideal gas law. Due to the low level of ionization at temperatures below $10000 \mathrm{~K}$, the ideal gas law can be applied without corrections as described in the literature. ${ }^{12,13}$ Heat loss by radiation is calculated using a multiband model with temperature dependent absorption coefficients reported in the literature. ${ }^{14}$ Since the emissivity of metals are higher than that of argon, ${ }^{15}$ the presence of metal atoms within the reactor can affect the radiative heat loss. However, the input concentrations of metal atoms in our experiments are very small (calculated as $5 \mathrm{ppm}$ based on the peristaltic pump flow rate, molarities of the aqueous solutions of metal salts, efficiency of the nebulizer, and the argon gas flow rates) compared to the values reported in the literature $(100 \mathrm{ppm}) .{ }^{15}$ We also performed a sensitivity analysis to verify that the radiative cooling due to the use of metals is weak to be neglected. The emission coefficients were changed by a factor ten and we observed that the resulting temperature distribution along the reactor varied only by a few of percent.

The small tube diameter $(37 \mathrm{~mm})$ and high viscosity of the hot plasma cause the fluid dynamic regime in the plasma torch to be laminar, whereas a local transitional regime occurs downstream of the ring flow injector due to the presence of the secondary injection of the ring flow and the increase of the hydraulic diameter. The turbulent k- $\varepsilon$ model for low Reynolds number was chosen to characterize this complex fluid dynamic regime. Figure 2 illustrates the simulated temperature profile and velocity field inside the plasma flow reactor. Lagrangian simulations allow the tracking of the injected material in the reactor and can be used to extract information about its temperature history. As an example, a streamline following a parcel of gas injected through the center of the plasma torch is highlighted in the figure. The flow field and axial temperature distribution show that the injected gas initially undergoes rapid cooling due to strong radiative heat loss at high temperatures. After the gas enters the plasma flow reactor tube, gas temperature drops rapidly due to mixing with the cold ring flow and radiative heat transfer becomes a second-order effect. Finally, when the temperature reaches approximately $1200 \mathrm{~K}$, convective heat transfer between the hot gas and the cold quartz wall $\left(20^{\circ} \mathrm{C}\right)$ causes a further steady decrease in temperature, which continues along the entire length of the reactor tube (see Fig. 3).

In Fig. 3, we show the temperature distribution of a parcel of gas as a function of residence time along the reactor at two different ring flow rates. Different temperature histories are obtained by varying the flow rate through the ring flow injector. This enables the evaluation of high temperature processes over a range of cooling histories spanning millisecond time scales. For example, according to our $14.5 \mathrm{l} / \mathrm{min}$ simulation, it takes approximately $100 \mathrm{~ms}$ for gas temperature to decrease to $1000 \mathrm{~K}$ whereas the temperature rapidly drops to the same value within $15 \mathrm{~ms}$ when the flow rate is increased to $19.3 \mathrm{l} / \mathrm{min}$. The plasma flow reactor can be operated with no ring flow gas injection, which further reduces the cooling rate in the high temperature core of the flow field (see discussion and results below).

\section{B. Temperature measurements}

Temperature measurements were performed along the central axis of the flow reactor using two different methods.
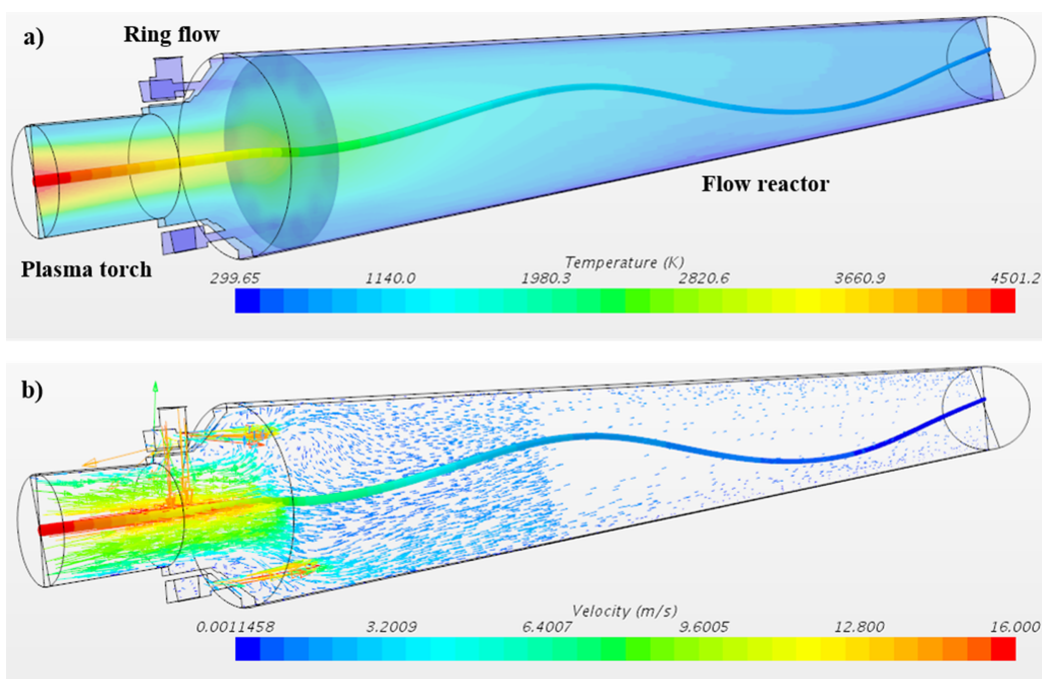

FIG. 2. Three-dimensional numerical representation of (a) temperature and (b) velocity fields inside the plasma flow reactor. The simulation results are shown for the first $40 \mathrm{~cm}$ section of the setup. The outermost, central, ring, and innermost flow rates were 14.5, 0, 12.1, and $1 \mathrm{l} / \mathrm{min}$, respectively. Changes in the density of velocity vectors in (b) are due to the use of an adaptive mesh in CFD simulations (i.e., a finer mesh is used for steep gradients). A streamline following a parcel of gas injected through the center of the plasma torch is highlighted in both figures. 


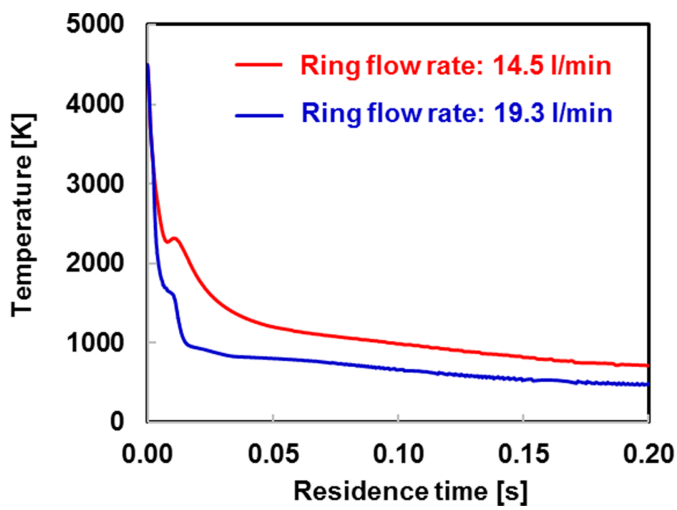

FIG. 3. The simulated temperature histories vs. residence time of a parcel of gas at two different ring flowrates.

Data were collected using a type-K thermocouple mounted on a moving stage. This method produces accurate data $( \pm 2 \mathrm{~K})$ for temperatures up to $1200 \mathrm{~K}$, above which radiation heat loss from the thermocouple bead becomes significant. This leads to a substantial increase in the temperature difference between the gas and the thermocouple. As a result, the thermocouple reading does not accurately represent the gas temperature above $1200 \mathrm{~K}$. Therefore, in the higher temperature region of the flow reactor, spectroscopic techniques were used to determine the temperature of the gas. Aqueous solutions of iron (III) nitrate or aluminum nitrate were injected through a nebulizer (Analytical West, AR35-1-FSS2E) at a rate of $0.2 \mathrm{ml} / \mathrm{min}$. Atomic (neutral iron) and molecular (aluminum monoxide) emission spectra were recorded at two locations before and after the ring flow injector, corresponding to distances of 2 and $5 \mathrm{~cm}$ from the RF coil, respectively. The spectra were recorded with a high resolution $(\mathrm{FWHM}=0.05 \mathrm{~nm})$ spectrometer (JY Horiba, HR 460). Measured emission spectra enabled the determination of gas temperature using analytical methods described by Lam et al. ${ }^{16}$ Atomic excitation temperatures were calculated from relative excited state populations described by Boltzmann's distribution function given by

$$
\ln \left(\frac{I_{i j} \lambda_{i j}}{g_{j} A_{i j}}\right)=-\frac{1}{k T} E_{j}+\ln \left(\frac{N h c}{Q}\right),
$$

where $I_{i j}$ is the intensity of the electronic transition between an excited level $j$ and a lower level $i, \lambda_{i j}$ is the wavelength at which the transition occurs, $A_{i j}$ is the Einstein transition probability of spontaneous emission, $E_{j}$ and $g_{j}$ are the energy and degeneracy of quantum level $j, N$ is the number density of the species involved, $k$ is the Boltzmann constant, $h$ is Planck's constant, $T$ is the temperature, $\mathrm{Q}(T)$ is the partition function of the atomic level system, and $c$ is the speed of light in vacuum. When the relative population $\left(I_{i j} \lambda_{i j} / g_{j} A_{i j}\right)$ is plotted versus the upper energy level $\left(E_{j}\right)$, the excitation temperature can be calculated from the slope of the line (slope $=-1 / k T)$.

Figure 4(a) is an emission spectra plot of neutral iron lines recorded at two different locations along the reactor using an iron nitrate analyte. The outermost, central, innermost, and ring flow rates were set to $14.5,0,1$, and $12.1 \mathrm{l} / \mathrm{min}$, respectively. As expected, emission intensities decrease with (a)

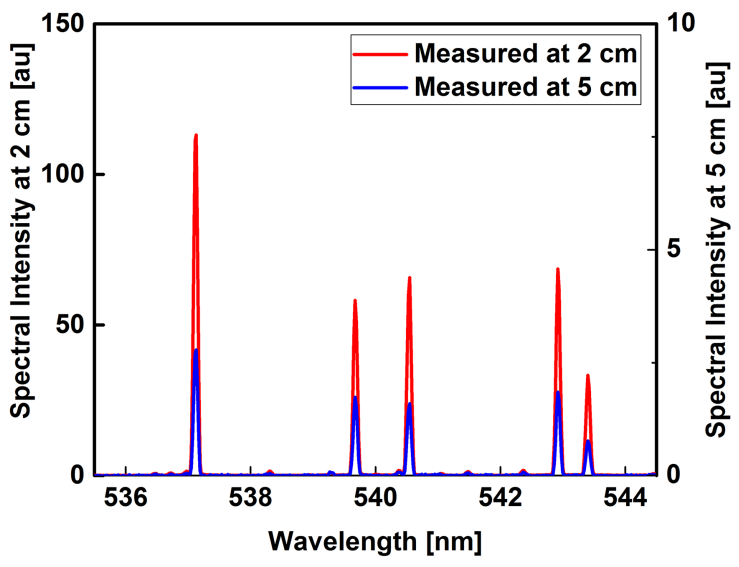

(b)

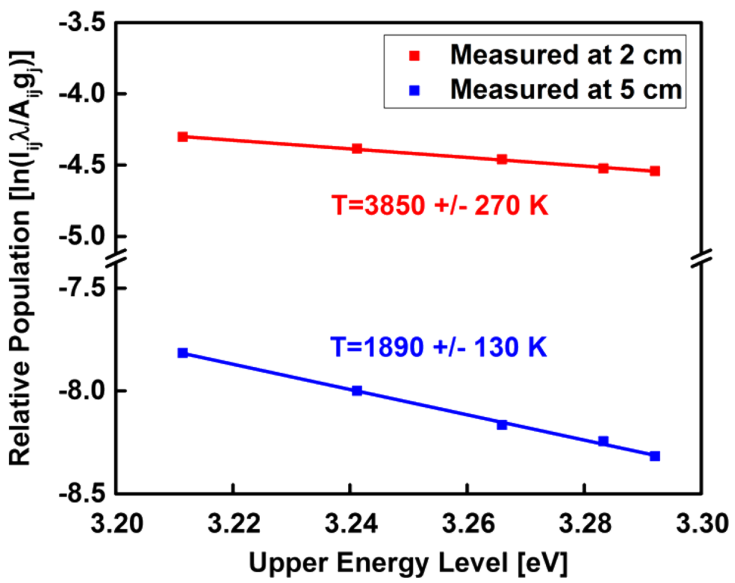

FIG. 4. (a) Comparison of measured emission spectra at two different axial locations: 2 and $5 \mathrm{~cm}$ from the end of the RF coil. (b) Corresponding temperature determinations from the slope of Boltzmann distribution line intensities.

increased distance away from the RF coil. In Fig. 4(b), we compare the temperatures calculated at those two locations using the measured line intensities $\left(I_{i j}\right)$ and the $\operatorname{NIST}^{17}$ reported values given in Table I. The results show the gas cooled rapidly from $3850 \mathrm{~K}$ at $2 \mathrm{~cm}$ to $1890 \mathrm{~K}$ at $5 \mathrm{~cm}$; the steep temperature gradient is due in part to the addition of cold argon gas from the ring flow injector.

According to Eq. (1) there is uncertainty in the calculation of temperature due to errors in the transition probabilities $\left(A_{i j}\right)$, upper energy levels $\left(E_{j}\right)$, and measured emission intensities $\left(I_{i j}\right)$. Also, an additional uncertainty in the calculation of temperature results from the least square fitting procedure that is used to determine the slope of the Boltzmann equation (slope $=-1 / k T)$. The accuracies of iron transition probabilities

TABLE I. Spectroscopic data for iron lines (used for temperature calculations ${ }^{17-19}$ ).

\begin{tabular}{llcr}
\hline $\begin{array}{l}\text { Neutral iron transition } \\
\text { wavelengths }(\mathrm{nm})\end{array}$ & $A_{i j}\left(10^{6} \mathrm{~s}^{-1}\right)$ & $E_{j}\left(\mathrm{~cm}^{-1}\right)$ & $g_{j}$ \\
\hline 537.1 & $1.1 \pm 0.029$ & $26339.696 \pm 0.001$ & 5 \\
539.7 & $0.3 \pm 0.0079$ & $25899.989 \pm 0.001$ & 9 \\
540.6 & $1.1 \pm 0.029$ & $26479.381 \pm 0.001$ & 3 \\
543.0 & $0.4 \pm 0.011$ & $26140.179 \pm 0.001$ & 7 \\
543.5 & $1.7 \pm 0.045$ & $26550.479 \pm 0.001$ & 1 \\
\hline
\end{tabular}


$\left(\sigma_{A_{i j}}= \pm 0.026 \%\right)$ and upper energy levels $\left(\sigma_{E_{j}}= \pm 0.001\right.$ $\mathrm{cm}^{-1}$ ) are reported in the literature ${ }^{18,19}$ and shown in Table I. The uncertainty in the measured intensity was assumed to be $\sigma_{I_{i j}}= \pm 1 \%$. The uncertainty in the slope of Eq. (1) was determined following the linear regression analysis discussion in the work of Bevington and Robinson. ${ }^{20}$ The resulting uncertainty in temperature was $\sigma_{T}= \pm 8 \%$.

A thorough review on how to determine translational gas temperature from rotational excitations of diatomic molecules is given by Bruggeman et al. ${ }^{21}$ Related studies ${ }^{16,22}$ discuss intrinsic deviations from local thermodynamic equilibrium based on differences in the excitation temperatures of atoms and molecules. These differences are due to variations in physical mechanisms of collisional energy transfers between atoms, molecules, and electrons. Since the accurate knowledge of translational gas temperatures is essential to confidently study high temperature chemical reactions, we conducted molecular emission measurements of aluminum monoxide using aluminum nitrate as the analyte and compared these results to those obtained from the atomic emissions of iron shown in Fig. 4.

In Figs. 5(a) and 5(b) we show measurements of the diatomic emission spectra of $\mathrm{AlO}$ recorded at the same locations and conditions as the Fe emission spectra discussed

(a)

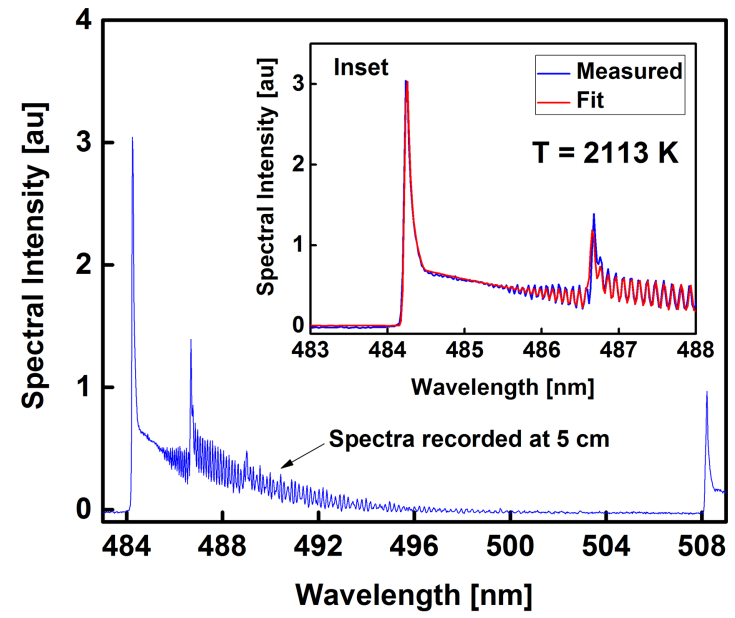

(b)

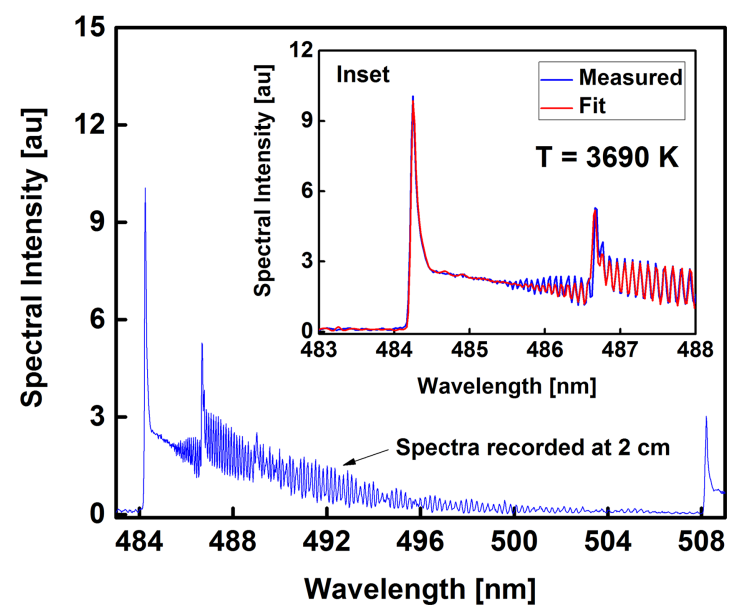

FIG. 5. Comparison of measured and calculated rovibronic spectra of $\mathrm{AlO}$ at two different axial locations away from the end of the RF coil: (a) $5 \mathrm{~cm}$ and (b) $2 \mathrm{~cm}$. above. The figure insets compare the measured spectra to the theoretical calculations obtained using a code provided in a recent study by Parigger et $a .^{23}$ We used this code to generate modeled fits to the measured $\mathrm{AlO}$ emission spectra. A Nelder-Mead nonlinear fitting algorithm is utilized in the code to compute temperatures. In the current study, this fitting program was used to determine the rotational temperature of the AlO molecule based on spectra for the rovibronic transition of $\mathrm{AlO}\left(\mathrm{B}^{2} \Sigma^{+} \rightarrow \mathrm{X}^{2} \Sigma^{+}\right)$. The comparison of the results in Figs. 4 and 5 indicate that the temperatures calculated for the Fe and $\mathrm{AlO}$ emission spectra using two independent methods are consistent to within the uncertainties $( \pm 10 \%)$ reported in the literature for the same type of temperature determination methods. ${ }^{16}$ This suggests that local thermodynamic equilibrium was achieved between the different degrees of freedom of the system (i.e., rotational and excitation temperatures). Similar emission measurements were conducted with different ring flow injector rates to quantify flow rate dependent temperatures. The results are compared to the fluid dynamic simulation predictions in Sec. III C. It was observed that the temperatures obtained from the atomic and molecular emissions followed a decreasing trend as the flow rate was increased.

In their studies, Lam et al. ${ }^{16}$ and Parigger et al. ${ }^{24}$ assumed uncertainties of $\pm 10 \%$ and $\pm 5 \%$ in temperature determination from measured emission spectra of $\mathrm{AlO}$ and $\mathrm{Fe}$, and AlO, respectively. In this study, we performed two types of calculations to estimate the error in the calculation of rotational temperature of $\mathrm{AlO}$ molecule. In the first calculation, we generated modeled fits to the measured spectra for different vibrational bands $(\Delta v=1,0,-1)$ of the rovibronic transition of $\mathrm{AlO}\left(\mathrm{B}^{2} \Sigma^{+} \rightarrow \mathrm{X}^{2} \Sigma^{+}\right)$. In the second calculation, we truncated the emission spectra for a given band and compared the fitting results. Variations in the calculated temperatures were less than $\pm 10 \%$.

\section{Comparison of experimental measurements and model predictions}

In Fig. 6 we plot a comparison between the experimentally measured temperatures and the predicted temperature profiles

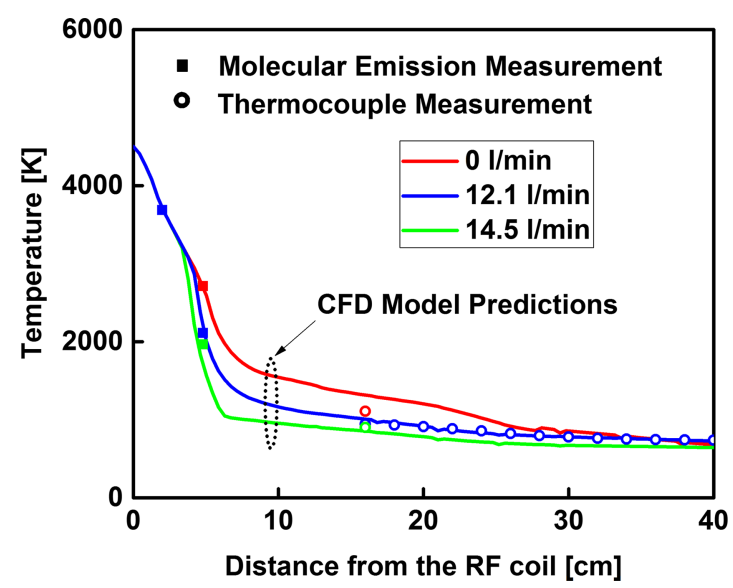

FIG. 6. Comparison of measured and modeled temperatures as a function of distance along the reactor. Lines are for model predictions and symbols are for measured values. Emission measurements were taken using an aluminum nitrate analyte. 
obtained from the computational fluid dynamics (CFD) model. Results are given for three different ring flow rates $(0,12.1$, and $14.5 \mathrm{l} / \mathrm{min})$. The other three flow rates were kept constant (outermost, central, and innermost flow rates were 14.5, 0, and $1 \mathrm{l} / \mathrm{min}$, respectively). The RF power was fixed at $715 \mathrm{~W}$. The experimental measurements shown at 2 and $5 \mathrm{~cm}$ were calculated from the emission spectra of $\mathrm{AlO}$, whereas the thermocouple was used to measure temperatures further downstream of the reactor ( $>16 \mathrm{~cm}$ from the RF coil). The computational model predicts a drop in temperature at the point where the gas expands from the plasma torch outlet (18 mm ID) into the flow reactor tube (37 $\mathrm{mm}$ ID). Cooling in this region, approximately $5 \mathrm{~cm}$ from the RF coil, is further enhanced by the addition of argon gas from the ring flow injector. The model shows a sharp decrease in temperature associated with increasing ring flow injection, in good agreement with the temperatures measured at $5 \mathrm{~cm}$ (see Fig. 6). The simulations overpredict the thermocouple temperature reading at $16 \mathrm{~cm}$ when the ring injector flow rate was reduced to zero (red curve in Fig. 6), but overall the CFD model is remarkably consistent with the measured temperatures and serves as a useful tool for inferring or predicting temperatures and flow fields inside the reactor across a wide range of conditions.

\section{APPLICATIONS OF THE PLASMA FLOW REACTOR}

The plasma flow reactor is well-suited for investigating dynamic chemical processes that involve rapid cooling in the 5000-1000 K temperature regime and on time scales ranging from a few milliseconds to several hundred milliseconds. Below, we discuss our preliminary work involving two distinct applications. The first study considers processes occurring during the condensation, nucleation, and growth of metal oxide particles. This work has applications to our understanding of the rapid condensation of matter. The second study examines chemical pathways following from plasma decomposition of a simple alkyne (acetylene: $\mathrm{C}_{2} \mathrm{H}_{2}$ ) and subsequent recombination reactions in an argon carrier gas. This work has relevance to the sequestration of waste products in fusion reactors. Whereas the second study involves only gas phase reactants, the first one requires aqueous solutions of metal salts. In both studies, the reaction mechanisms are expected to occur on rapid time scales wherein kinetic processes dominate.

\section{A. Condensation and growth of metal oxides}

Commercial ICP-OES and ICP-MS systems routinely use an analytical nebulizer to introduce liquid samples to the plasma torch for the analysis of metal concentrations in solution. We utilized this technology to investigate the formation of metal oxide particles in the plasma flow reactor when relatively concentrated metal solutions are injected into the plasma flow reactor. The solution is thermally decomposed into its constituent elements as it passes through the plasma, and excess oxygen from the water reacts with the metal atoms to form oxides downgradient from the plasma torch. Optical spectroscopy provides in situ data regarding the time evolution and temperature of the metal oxide formation. In addition, particles that form during condensation are sampled using a collection probe described in Sec. II of this paper. The collection substrate consisted of either a silicon wafer $(5 \mathrm{~mm} \times 5 \mathrm{~mm})$ or a TEM grid attached to the end of the probe. Collected particles were characterized using electron microscope techniques (SEM and TEM).

We tested this application using an aqueous solution of iron and aluminum nitrate containing $1.5 \times 10^{20}$ atoms $/ \mathrm{ml}$ of each metal $(13.9 \mathrm{mg} / \mathrm{ml} \mathrm{Fe} ; 6.7 \mathrm{mg} / \mathrm{ml} \mathrm{Al})$. The nebulizer delivers a steady-state input of $0.2 \mathrm{ml} / \mathrm{min}$ of solution to the plasma torch. Both iron and aluminum monoxide rovibronic spectra were observed as shown in Fig. 7. The spectra were recorded at $5 \mathrm{~cm}$ downstream of the RF coil. The emission spectra were assigned to $\mathrm{AlO}$ and $\mathrm{FeO}$ molecules based on literature data. ${ }^{25,26}$ The atomic emission lines of iron between 520 and $560 \mathrm{~nm}$ were omitted in Fig. 7 to clearly show the molecular spectra. The temperature of the Fe atoms was determined to be $1740 \mathrm{~K}( \pm 10 \%)$ based on the Fe emission lines, which is consistent with an earlier temperature measurement made at the same position along the flow reactor axis in the absence of aluminum [see Fig. 4(b)].

We used a silicon wafer to collect aluminum oxide particles $16 \mathrm{~cm}$ downgradient from the RF coil, see Fig. 8(a). The wafer was carbon coated before performing imaging to create a conductive layer to prevent charging and thus to improve the secondary electron imaging. A particle size distribution was observed on the wafer, ranging from tens of nanometers up to an agglomerate of almost a micron in size. Figure 8(b) is our qualitative energy dispersive spectroscopy (EDS) plot of the 925-nm particle shown in Fig. 8(a). The EDS results indicate an $\mathrm{Al}: \mathrm{Fe}$ atomic ratio of $\sim 60: 1$, implying the formation of aluminum oxide was strongly favored over iron oxide at this location in the flow reactor. Since the electron beam has a finite size and the particle is relatively small, the elemental analysis also includes silicon and carbon peaks from the underlying substrate. The $\mathrm{Al}: \mathrm{Fe}$ ratio of the particles was observed to decrease with increasing distance from the plasma, implying that Fe-rich particle formation is favored at lower temperatures.

In Fig. 9(a), we provide a bright-field TEM image of a 225-nm spherical particle that was collected on a TEM

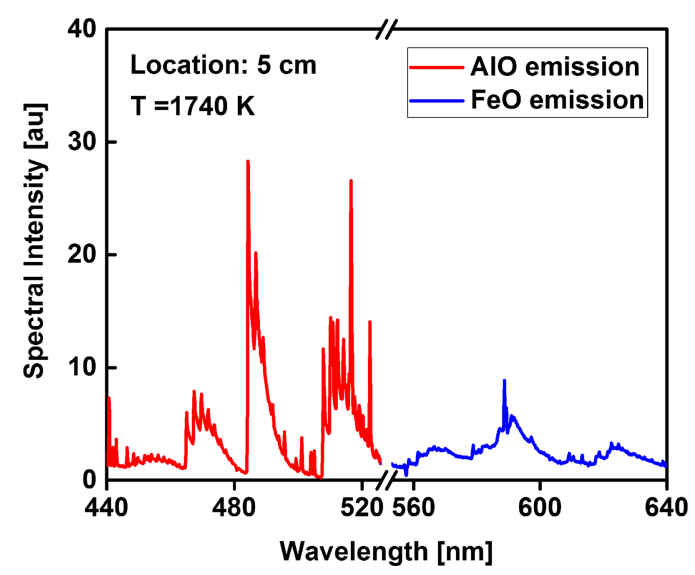

FIG. 7. Broad molecular emission spectra of $\mathrm{AlO}$ and FeO. A mixture of aluminum nitrate $\left[\mathrm{Al}\left(\mathrm{NO}_{3}\right)_{3} \cdot 9 \mathrm{H}_{2} \mathrm{O}\right]$ and iron nitrate $\left[\mathrm{Fe}\left(\mathrm{NO}_{3}\right)_{3} \cdot 9 \mathrm{H}_{2} \mathrm{O}\right]$ was used as the analyte. 

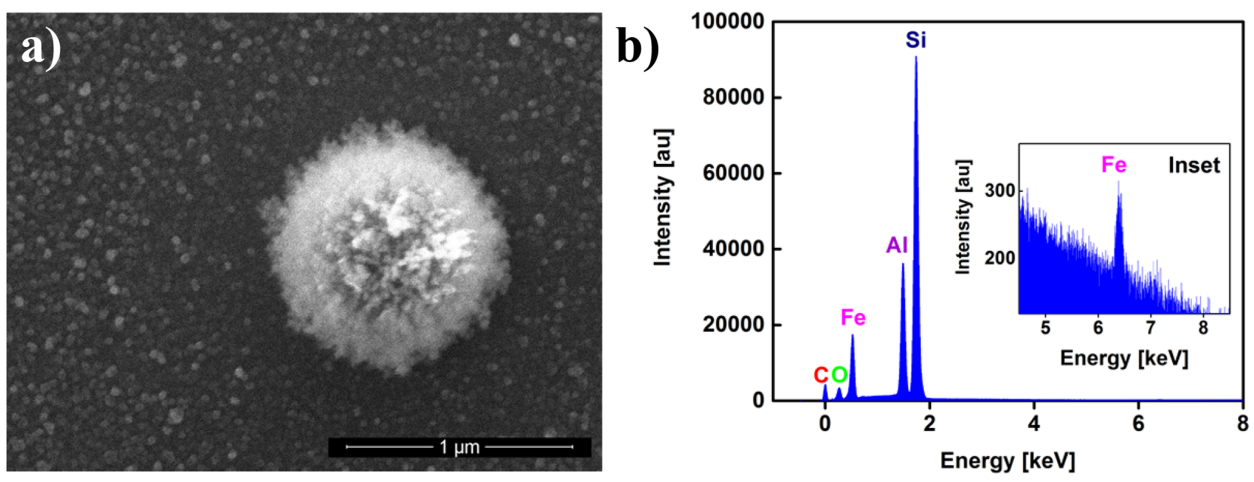

FIG. 8. (a) An SEM image of a recovered metal oxide and (b) elemental composition analysis based on $\mathrm{x}$-ray energy dispersive spectroscopy.

grid at the same location as the particle shown in Fig. 8(a). A high-resolution image of this particle [Fig. 9(b)] shows it is crystalline and an agglomeration of many smaller $(5-10 \mathrm{~nm})$ particles. EDS analysis gave a composition of 63 at. \% oxygen, 36 at. $\%$ aluminum, and $<1$ at. \% iron; no substrate interference was encountered in this case. Electron diffraction analysis [Fig. 9(c)] revealed that crystal structure of the particle matches the cubic eta- $\mathrm{Al}_{2} \mathrm{O}_{3}$ crystal (space group: $\mathrm{Fd}-3 \mathrm{~m}$ : lattice parameter: $\mathrm{a}=7.906 \AA) .{ }^{27}$ Further analysis on the structure of the iron oxide particles will be the subject of a future study.

\section{B. Chemical kinetics investigations}

In another set of experiments, the plasma flow reactor was used to examine the non-combustion decomposition and recombination kinetics of simple hydrocarbons. Chemical kinetics simulations ${ }^{28}$ show that acetylene $\left(\mathrm{C}_{2} \mathrm{H}_{2}\right)$ passing through an argon plasma will initially decompose, but some fraction of the breakdown products will recombine to form new $\mathrm{C}_{2} \mathrm{H}_{2}$ molecules when the temperature decreases to $\sim 1000 \mathrm{~K}$. In contrast, calculations based on chemical equilibrium indicate that no $\mathrm{C}_{2} \mathrm{H}_{2}$ recombination should occur under these conditions. We used the plasma flow reactor and input a small amount of acetylene ( $\sim 536 \mathrm{ppm})$ through the innermost annulus of the plasma torch via a digital flow controller (Omega, FMA 2616A) in order to acquire spectroscopic data that would allow us to resolve this inconsistency. This topic has relevance to chemical processes that may occur in fusion energy research reactors.
The detection of recombined acetylene downstream of the reactor was accomplished through laser diagnostics. Figure 1 includes a schematic of the laser system. We used a laser diode (Nanoplus DFB ICL) with an approximately 3- $\mu \mathrm{m}$ wavelength continuous-wave beam. After the laser beam passes through the flow reactor, it is directed onto a thermoelectrically cooled MCT type detector (HgCdTe, Vigo Systems PVI-3TE-4). Since the laser power was relatively small $(<3 \mathrm{~mW})$, a neutral density filter and a band pass filter (Northumbria Optical Coatings, CWL $2998 \mu \mathrm{m}$, BW 0.93\%) were used to attenuate and minimize unwanted optical interference on the detector due to high temperature gas emissions. The reference $\left(I_{\text {ref }}\right)$ and sample $\left(I_{\text {sample }}\right)$ intensities were recorded on the detector when pure argon and $536 \mathrm{ppm}$ of acetylene were flowing through the reactor, respectively. The attenuation of light intensity due to the absorption of acetylene enabled the quantification of its concentration using the Beer-Lambert law,

$$
\alpha_{v}=-\ln \left(\frac{I_{\text {Sample }}}{I_{\text {ref }}}\right)_{v}=\sigma(v, T, P) \frac{P_{\text {tot }}}{R T} \chi L,
$$

where $\alpha_{v}$ is the absorbance, $\sigma\left(\mathrm{cm}^{2} / \mathrm{mol}\right)$ is the absorption cross section, $P_{\text {tot }}(\mathrm{atm})$ is the total pressure within the system, and $T(\mathrm{~K})$ is the temperature of the gas. $L(\mathrm{~cm})$ is the optical path length (plasma flow reactor inner diameter is $37 \mathrm{~mm}$ ) and $\chi$ is the mole fraction of the absorbing species. Stranic and Hanson ${ }^{29}$ characterized the absorption cross sections of $\mathrm{C}_{2} \mathrm{H}_{2}$ using a shock tube at various conditions $(1070 \mathrm{~K}<\mathrm{T}$ $<1720 \mathrm{~K}$ and $0.8 \mathrm{~atm}<\mathrm{P}<4.0 \mathrm{~atm})$. The current study
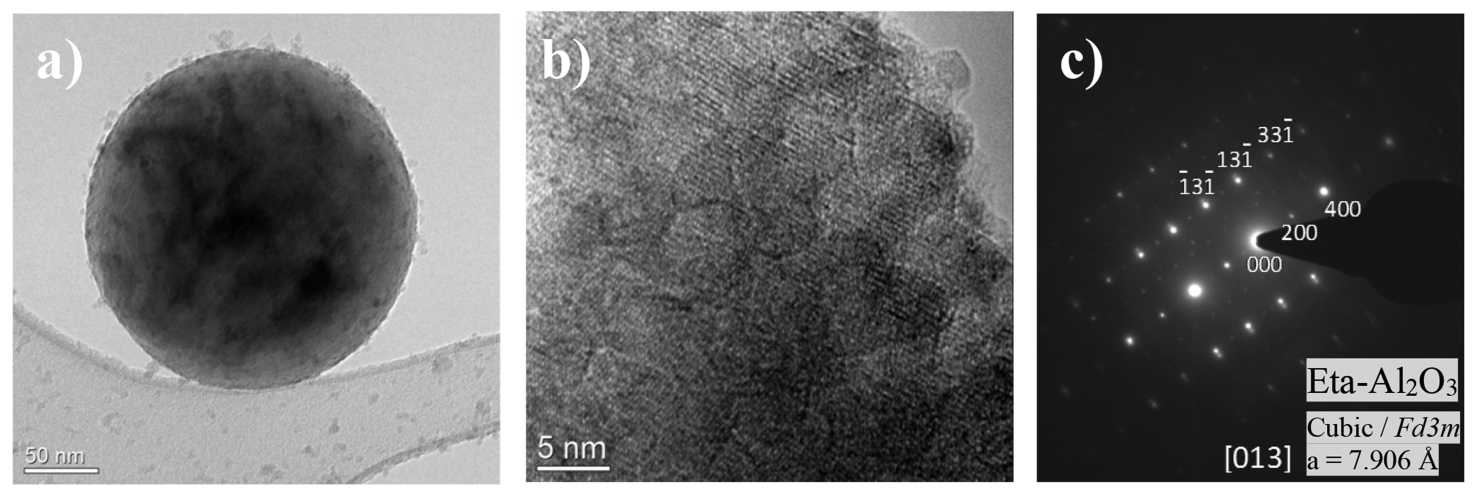

FIG. 9. (a) The bright-field TEM image of a metal oxide particle, (b) high resolution TEM image, and (c) selected-area electron diffraction pattern of [013] zone axis of eta- $\mathrm{Al}_{2} \mathrm{O}_{3}$ 


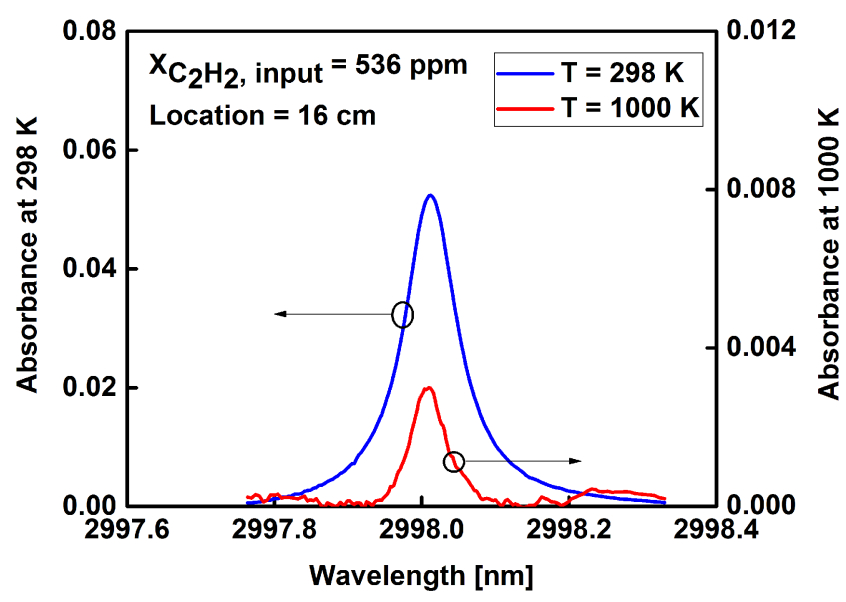

FIG. 10. Infrared spectroscopy measurements centered at $2998 \mathrm{~nm}$. Measurements are shown for two temperatures $298 \mathrm{~K}$ (ICP off) and $1000 \mathrm{~K}$ (ICP on), at $16 \mathrm{~cm}$ downstream of the RF coil.

uses a very similar optical arrangement as well as the reported values of the cross sections to calculate mole fractions from our measured intensities. The measured intensities reported in this paper were an average of 1000 scans.

Figure 10 is a plot of line of sight laser absorption measurements taken at $16 \mathrm{~cm}$ away from the RF coil. The infrared spectra were recorded for two cases. For the first case, the $\mathrm{RF}$ generator was off, and therefore the temperature within the reactor was room temperature $(298 \mathrm{~K})$. In this case, the measured absorbance displayed on the left y-axis of Fig. 10 resulted in the expected mole fraction of $536 \mathrm{ppm}$ (this is the expected concentration based on the flow rate settings on the digital flow controllers). For the second case, the RF generator was on and the temperature at $16 \mathrm{~cm}$ away from the RF coil was $1000 \mathrm{~K}$. The mole fraction of $\mathrm{C}_{2} \mathrm{H}_{2}$ (calculated using the measured absorbance shown on the right y-axis of Fig. 10) was $195 \mathrm{ppm}$. This indicates that $35 \%$ of the initially input acetylene was recombined after decomposition at the argon plasma. More data and further details with comparisons to chemical kinetics simulations will be discussed in a separate paper.

Based on the Beer lambert law [Eq. (2)], the uncertainty of the mole fraction measurements is dependent on the errors in the measurements of absorbance, absorption crosssection, temperature, pressure, and path length. The uncertainties of the acetylene absorption cross sections were reported as $\sigma_{\sigma}= \pm 3 \%$ around $1000 \mathrm{~K} .{ }^{29}$ The accuracy of the type K thermocouple is $\sigma_{T}= \pm 0.75 \%$ of the reading. Our flow controllers are equipped with absolute pressure sensors and their error is $\sigma_{P}= \pm 0.0544 \mathrm{~atm}$. The errors in path length and absorbance are taken as $\sigma_{L}= \pm 0.5 \mathrm{~mm}$ and $\sigma_{\alpha}= \pm 1 \%$. The resulting uncertainty in mole fraction measurement is $\sigma_{\chi}= \pm 7 \%$.

We also performed emission measurements to check the decomposition products of $\mathrm{C}_{2} \mathrm{H}_{2}$. The emission intensity follows a Boltzmann distribution [see Eq. (1)] and therefore, it is dependent on the number density and temperature. If gas temperature stays constant at different analyte flow rates, then the emission intensity will vary according to the changes in the number densities of species. Therefore, gas temperatures were measured based on $\mathrm{C}_{2}$ rotational lines recorded at $5 \mathrm{~cm}$ away from the RF coil for various $\mathrm{C}_{2} \mathrm{H}_{2}$ input flow rates. We observed that changes in temperature were well below the uncertainties of the measurements. Atomic carbon intensities were also recorded at different analyte flow rates further upstream of the reactor and we saw a one-to-one match between the ratios of carbon emission intensities and of $\mathrm{C}_{2} \mathrm{H}_{2}$ analyte flow rates (i.e., when the input amount of $\mathrm{C}_{2} \mathrm{H}_{2}$ was doubled, the carbon emission intensity doubled as well). As a result, we assume all $\mathrm{C}_{2} \mathrm{H}_{2}$ is thermally decomposed as it passes through the argon plasma. In addition, complete decomposition of analyte $\left(\mathrm{C}_{2} \mathrm{H}_{2}\right)$ was confirmed through chemical kinetics calculations using Chemkin-Pro. ${ }^{30}$ Further details with measurement results will be given in a separate paper.

\section{CONCLUSIONS}

We have developed a novel steady-state plasma flow reactor and have characterized its performance through computational fluid dynamics simulations and in situ temperature measurements obtained from emission spectra and thermocouple data. The reactor is comprised of an inductively coupled argon plasma torch that is attached to a flow reactor tube using an adapter (ring flow injector). The reactor offers a flexible experimental platform for investigating high temperature (1000-5000 K) gas phase chemical processes occurring on rapid time scales (up to $100 \mathrm{~ms}$ ) at atmospheric pressures. The ring flow injector permits the fluid dynamic conditions inside the flow reactor to be adjusted using either inert or reactive gases.

The flow reactor performance was studied using two types of starting materials: (1) aqueous solutions of metal salts and (2) dilute hydrocarbon gas. In both applications, optical spectroscopy measurements (visible and/or mid-IR) were used to collect in situ data to characterize the gas phase chemistry. The steady state nature of the flow reactor enables the chemical evolution of the system to be monitored through the collection of spectral data at different positions along the length of the reactor tube. Emission spectra also allow for the simultaneous determination of temperature for selected materials.

For experiments involving aqueous metal salt solutions, we demonstrated the feasibility of in situ particle collection and post experimental characterization of particles using scanning and transmission electron microscopy. For example, aluminum oxide particles up to $1 \mu \mathrm{m}$ in diameter appear to form through the agglomeration of nano-crystalline $\mathrm{Al}_{2} \mathrm{O}_{3}$ particles generated from an aqueous aluminum nitrate starting material. This application can be used to study particle nucleation, condensation, and growth processes.

In a second type of experiment, hydrocarbon gas (acetylene) was decomposed in the plasma and absorption spectroscopy was used to study the recombination kinetics of reactions. This work was directed toward understanding chemical reactions associated with the cyclic heating and rapid cooling in fusion energy chambers. However, it has a broader application to expanding our understanding of the time dependent gas phase chemical reactions. Steady state monitoring of reaction progress over a range of cooling histories using infrared absorption spectroscopy allows us to determine the concentration of chemical species as a function of residence 
time along the reactor. The concentration time-histories of chemical species can be used to measure high-temperature reaction rate coefficients, which are generally difficult to obtain. In this way, we can also offer new insights into the transition from kinetically controlled to equilibrium chemical mechanisms for different high temperature applications.

\section{ACKNOWLEDGMENTS}

Funding was provided by Laboratory Directed Research and Development (LDRD) Grant No. 14-ERD-077 (M. Armstrong, PI) and 16-ERD-008 (T. Rose, PI). This work was performed under the auspices of the U.S. Department of Energy by Lawrence Livermore National Laboratory under Contract No. DE-AC52-07NA27344.

${ }^{1}$ M. I. Boulos, Pure Appl. Chem. 57, 1321 (1985).

${ }^{2}$ M. Thompson, Handbook of Inductively Coupled Plasma Spectrometry (Springer Science \& Business Media, 2012).

${ }^{3}$ V. Godyak, J. Phys. D: Appl. Phys. 46, 283001 (2013).

${ }^{4}$ M. A. Cappelli, T. G. Owano, and C. H. Kruger, J. Mater. Res. 5, 2326 (1990).

${ }^{5}$ K. S. Kim, A. Moradian, J. Mostaghimi, Y. Alinejad, A. Shahverdi, B. Simard, and G. Soucy, Nano Res. 2, 800 (2009).

${ }^{6}$ M. A. Mueller, T. J. Kim, R. A. Yetter, and F. L. Dryer, Int. J. Chem. Kinet. 31, 113 (1999).

${ }^{7}$ R. K. Hanson and D. F. Davidson, Prog. Energy Combust. Sci. 44, 103 (2014).

${ }^{8}$ B. Koroglu, O. M. Pryor, J. Lopez, L. Nash, and S. S. Vasu, Combust. Flame 164, 152 (2016).

${ }^{9}$ S. B. Punjabi, S. N. Sahasrabudhe, S. Ghorui, A. K. Das, N. K. Joshi, D. C. Kothari, A. A. Ganguli, and J. B. Joshi, AIChE J. 60, 3647 (2014).

${ }^{10}$ W. L. T. Chen, J. Heberlein, and E. Pfender, Plasma Chem. Plasma Process. 16, 635 (1996).

${ }^{11}$ T. Hoshino, K. Mito, A. Nagashima, and M. Miyata, Int. J. Thermophys. 7, 647 (1986)
${ }^{12}$ W. Lochte-Holtgreven, Plasma Diagnostics (North Holland Publishing Company, 1968).

${ }^{13}$ H. R. Griem, Phys. Rev. 128, 997 (1962).

${ }^{14}$ V. Aubrecht, M. Bartlova, and N. Bogatyreva, in 29th International Conference on Phenomena in Ionized Gases, 2009, available: Https://Www.researchgate.net/Publication/267557923_Radiation_properties_of_argon_thermal_plasma_in_various_spectral_regions.

${ }^{15}$ J. Menart and S. Malik, J. Phys. D: Appl. Phys. 35, 867 (2002).

${ }^{16}$ J. Lam, V. Motto-Ros, D. Misiak, C. Dujardin, G. Ledoux, and D. Amans, Spectrochim. Acta, Part B 101, 86 (2014).

${ }^{17}$ A. Kramida, Y. Ralchenko, and J. Reader, and NIST ASD Team, NIST Atomic Spectra Database (Version 5.4), [Online], National Institute of Standards and Technology, Gaithersburg, MD, 2016, available: Http://Physics.nist.gov/Asd [Mon May 08 2017].

${ }^{18}$ D. E. Blackwell, A. D. Petford, and M. J. Shallis, Mon. Not. R. Astron. Soc. 186, 657 (1979).

${ }^{19}$ G. Nave, S. Johansson, R. C. M. Learner, A. P. Thorne, and J. W. Brault, Astrophys. J., Suppl. Ser. 94, 221 (1994).

${ }^{20} \mathrm{P}$. R. Bevington and D. K. Robinson, Data Reduction and Error Analysis for the Physical Sciences (McGraw-Hill, New York, 1992).

${ }^{21}$ P. J. Bruggeman, B. Sadeghi, D. C. Schram, and V. Linss, Plasma Sources Sci. Technol. 23, 023001 (2014).

${ }^{22}$ A. Sola, M. D. Calzada, and A. Gamero, J. Phys. D: Appl. Phys. 28, 1099 1110 (1995)

${ }^{23}$ C. G. Parigger, A. C. Woods, D. M. Surmick, G. Gautam, M. J. Witte, and J. O. Hornkohl, Spectrochim. Acta, Part B 107, 132 (2015).

${ }^{24}$ C. Parigger, D. H. Plemmons, J. O. Hornkohl, and J. W. L. Lewis, J. Quant. Spectrosc. Radiat. Transfer 52, 707 (1994).

${ }^{25}$ M. Gaft, L. Nagli, N. Eliezer, and Y. Groisman, Spectrochim. Acta, Part B 110, 56 (2015).

${ }^{26}$ C. G. Parigger and J. O. Hornkohl, Spectrochim. Acta, Part A 81, 404 (2011).

${ }^{27}$ K. Shirasuka, H. Yanagida, and G. Yamaguchi, J. Ceram. Assoc., Japan 84(976), 610-613 (1976).

${ }^{28}$ M. Mehl, M. Armstrong, J. Zaug, J. Crowhurst, H. Radousky, and E. Stavrou, in 58th Annual Meeting of the APS Division of Plasma Physics (2016), Vol. 61, Number 18, Presentation: PO7.00012, available: http:// meetings.aps.org/Meeting/DPP16/Session/PO7.12.

${ }^{29}$ I. Stranic and R. K. Hanson, J. Quant. Spectrosc. Radiat. Transfer 142, 58 (2014).

${ }^{30}$ CHEMKIN-PRO 15131, Reaction Design, San Diego, 2013. 